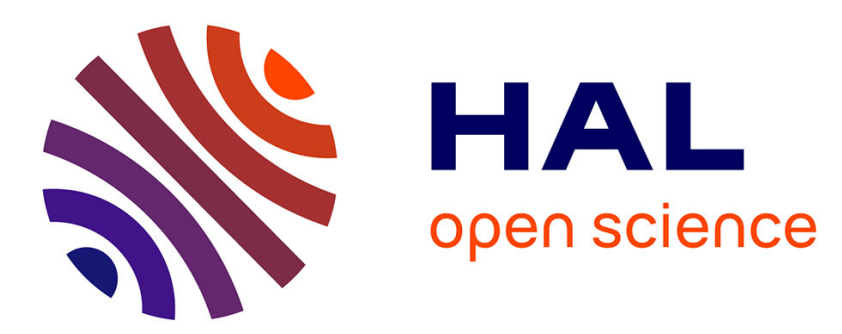

\title{
Analyse de référents de l'activité de co-design dans un contexte de Réalité Mixte
}

Stéphane Safin, Annemarie Lesage, Anne-Marie Hébert, Gokce Kinayoglu, Tomas Dorta

\section{- To cite this version:}

Stéphane Safin, Annemarie Lesage, Anne-Marie Hébert, Gokce Kinayoglu, Tomas Dorta. Analyse de référents de l'activité de co-design dans un contexte de Réalité Mixte. 25ème conférence francophone sur l'Interaction Homme-Machine, IHM'13, AFIHM, Nov 2013, Bordeaux, France. $10.1145 / 2534903.2534908$. hal-00875456v2

\section{HAL Id: hal-00875456 https://hal.inria.fr/hal-00875456v2}

Submitted on 6 Nov 2013

HAL is a multi-disciplinary open access archive for the deposit and dissemination of scientific research documents, whether they are published or not. The documents may come from teaching and research institutions in France or abroad, or from public or private research centers.
L'archive ouverte pluridisciplinaire HAL, est destinée au dépôt et à la diffusion de documents scientifiques de niveau recherche, publiés ou non, émanant des établissements d'enseignement et de recherche français ou étrangers, des laboratoires publics ou privés. 


\title{
Analyse de référents de l'activité de co-design dans un contexte de Réalité Mixte
}

\author{
Stéphane Safin ${ }^{1}$, Annemarie Lesage ${ }^{2}$, Anne-Marie Hébert ${ }^{3}$, Gokce Kinayoglu ${ }^{4}$ et Tomás Dorta ${ }^{5}$ \\ ${ }^{1-5}$ Hybridlab, Université de Montréal \\ 5155 Decelles, Pavillon JA Bombardier, bureau 4027 \\ Montréal, Québec, H3T 2B1 +1(514)340-4711x2207 \\ ${ }^{1}$ Laboratoire C3U Paragraphe, Université Paris 8, France \\ stephane.safin@gmail.com ${ }^{1}$, annemarie.lesage@umontreal.ca ${ }^{2}$, anne-marie.hebert.2@umontreal.ca ${ }^{3}$, \\ gokce.kinayoglu@montreal.ca ${ }^{4}$, tomas.dorta@umontreal.ca ${ }^{5}$
}

\section{RESUME}

Cette étude porte sur l'analyse de référents de l'activité de co-design dans un contexte de réalité mixte à travers l'utilisation d'un dispositif de co-design installé au sein même de l'espace à concevoir ; le contexte de design offrant des représentations réelles et virtuelles du même espace à concevoir. Notre objectif de recherche est de comprendre comment ces deux représentations réelles et virtuelles sont utilisées comme ressources à des fins de co-design dans un cadre multidisciplinaire. Pour ce faire, une analyse des gestes de pointage effectués par les participants a été conduite. Nos résultats soulignent une complémentarité du virtuel et réel en début de processus pour soutenir le travail de co-design et la mise en relation des éléments de design avec des repères réels. Ensuite, les représentations virtuelles sont majoritairement utilisées pour soutenir le design et la construction d'un common ground ce qui met en exergue les représentations virtuelles immersives de cette étude comme suffisantes pour concevoir.

\section{ABSTRACT}

This paper focuses on referents used in co-design in a Mixed Reality context. A co-design device was installed inside a space to be redesigned, thus offering at once real and virtual representations of the space. Our goal is to understand how the real and virtual representations are used as resource in multidisciplinary co-design. An analysis of pointing gestures done by participants was conducted. Our results underscore complementarities at first between the real and the virtual, as design elements are matches to markers in the real space. Then virtual representations are mostly used to support the design and the construct of a common ground, highlighting the ability of the virtual representations to support design.

\section{Mots Clés}

Réalité mixte; co-design; multidisciplinarité; immersion; pointage.

(C) ACM, 2013. This is the author's version of the work. It is posted here by permission of ACM for your personal use. Not for redistribution. The definitive version was published in Actes de la $25^{\text {ième }}$ conférence francophone sur l'Interaction Homme-Machine, 2013.

http://dx.doi.org/10.1145/2534903.2534908

\section{ACM Classification Keywords}

H.5.1 [Multimedia Information Systems] Artificial, augmented, and virtual realities. H.5.3 [Group and Organization Interface] Computer-supported cooperative work

\section{INTRODUCTION}

De plus en plus, la conception de produits, services ou bâtiments nécessite une collaboration étroite entre plusieurs corps de métiers. De nombreux outils et méthodes existent pour faciliter la collaboration dans des projets d'envergure, pour faciliter l'émergence de concepts créatifs collectifs, la prise de décision consensuelle, le partage de représentations et l'évaluation des solutions proposées.

Plus récemment, on voit aussi émerger deux besoins ou tendances en parallèle. La première consiste à intégrer la collaboration dans les étapes très précoces de la conception, c'est-à-dire la conception dite préliminaire ou idéation. Le second besoin est relatif au profil des personnes à faire intervenir dans l'idéation: clients, utilisateurs, peuvent ainsi être amenées à interagir ensemble autour des concepts, idées et représentations précédant la production d'un nouveau produit ou service, ou la construction d'un nouveau bâtiment.

Il convient dès lors de fournir à ces utilisateurs designers, architectes mais aussi maître d'ouvrages, ingénieurs ou autres - des outils de représentation appropriés pour interagir ensemble autour de l'objet de conception.

\section{LE DESIGN COLLABORATIF}

La conception collaborative nécessite trois classes d'activités pour être menée à bien [5] :

- des activités centrées sur le contenu (tel le design d'un espace) ;

- des activités centrées sur le processus (tel que la collaboration ou design multidisciplinaire en simultanée) ;

- des activités de gestion de l'interaction (activités d'encadrement, tel l'orchestration de rencontres en plénière et petits groupes).

Pour nourrir les activités centrées sur le contenu ou sur le processus, il est nécessaire de construire des images mentales de l'objet ou du lieu à concevoir, supportées 
essentiellement par les différents modes de représentations disponibles (plans, maquettes, modèles numériques, etc.) [20]. Or, dans les étapes initiales de la conception, ces images mentales sont assez floues, et les modes de représentations utilisés, notamment par le biais des dessins à main levée, sont encore imprécis [14].

De plus, et c'est particulièrement le cas pour soutenir les approches multidisciplinaires, il apparait que les compétences d'expression graphiques sont principalement l'apanage des designers et architectes formés à l'utilisation des modes de représentation classiques telle que l'esquisse à main levée ou le maquettage (physique), et des modes d'expression numériques telles que la modélisation 3D ou le dessin assisté par ordinateur (CAO). Ils sont aussi plus à même de se former à la création d'une image mentale de l'objet à concevoir, sans la représenter [3].

De plus, les plans, représentations codifiées, ne sont pas faciles à comprendre par les non-designers (du fait d'un haut niveau d'abstraction). Des modèles physiques ou virtuels permettent de se faire une représentation plus appropriée des composantes d'un espace, notamment des ambiances [21].

Dans cet article, nous présentons nos observations lors d'un atelier de design collaboratif multidisciplinaire lié à une problématique architecturale ayant fait appel à un l'environnement technologique immersif, le Hybrid Ideation Space (HIS). L'expérience visait à mettre en place une situation de réalité mixte pour supporter le processus de co-design : l'environnement immersif, dans l'étude présentée ici, a été inséré au cœur de l'espace réel faisant l'objet du travail de conception, et ouvert sur celui-ci (l'écran à $360^{\circ}$ est ouvert sur environ $120^{\circ}$ ). Les participants voient donc en même temps l'espace réel et le modèle virtuel de cet espace. C'est justement le rapport entre ces deux espaces, les participants étant tous plongés à la fois dans l'espace réel et virtuel, qui constitue l'originalité du dispositif expérimental et le cœur de notre étude.

Nous avons ici cherché à comprendre comment les environnements réel et virtuel fournissent des opportunités et des ressources pour la construction d'un référentiel commun, d'une vision partagée du problème et d'une orientation vers l'action collective et multidisciplinaire dans la co-conception.

\section{REALITE MIXTE}

Contrairement aux environnements entièrement médiatisés qui se proposent comme substituts de la réalité, la masquant et la replaçant par une expérience numérique, la réalité mixte (RM) consiste en une utilisation conjointe de ressources issues du monde réel (réalité physique) et du monde numérique (modèles, environnements virtuels, etc.) [17]. Il existe de nombreuses déclinaisons du concept qui s'inscrivent le long d'un «continuum de virtualité » dépendant notamment du caractère numérique ou réel de l'objet, de l'activité ou de l'environnement et l'activité $[10,17]$.

La réalité augmentée (RA), cas particulier de le RM, est plus spécifiquement définies par trois caractéristiques: 1) Elle associe du contenu réel et virtuel; 2) elle permet l'interactivité en temps réel; 3) le contenu virtuel se superpose à la réalité, associé et mis en contexte au sein de l'environnement réel [2]. La RM et la RA s'inscrivent dans un paradigme écologique de la perception et de la présence puisque les interactions entre le corps et l'espace font partie intégrante du cadre de l'interaction.

Anders [1] propose trois principes pour concevoir des interfaces hybrides fusionnant le virtuel et le réel de sorte qu'ils permettent l'interaction de plusieurs acteurs:

- Les attentes situées, ancrées dans le corps (embodied) doivent être confirmées par la rétroaction que donne le numérique ;

- Le contexte est une considération importante car il indique le type d'interaction possible dans cet espace ;

- Il doit y avoir une correspondance entre les espaces réels et virtuels pour que la réalité mixte soit opérante.

Anders n'a pas étudié de cas exemplifiant l'application de ces principes. Gibson [12] a déjà établi que la perception visuelle s'appuie sur le mouvement (l'effet de parallaxe). Donc on peut supposer qu'un utilisateur obéissant aux principes d'Anders aura initialement un mouvement d'aller-retour entre l'espace réel et virtuel, ayant pour but de consolider sa perception de l'espace mixte.

\section{LA NOTION D'AFFORDANCE}

L'usage qu'ont fait les participants des espaces immersifs réels et virtuels a soulevé des considérations quant à l'affordance de ces espaces (ou de la représentation de ces espaces) pour soutenir la conception. La notion d'affordance a été définie initialement par Gibson [13] et subséquemment par Norman [18, 19]. Gibson définit une affordance comme un état physique permettant une action spécifique (ex. une table permet à une assiette d'être soutenue), c'est-àdire, qu'elle est la possibilité d'une action. Norman définit une affordance en terme de propriétés réelles et perçues, c'est-à-dire si une propriété est perçue comme permettant une action, elle est une affordance. McGrenere et Ho [16] établissent la différence entre les deux définitions à ce que Norman inclut à la possibilité d'une action, la manière dont cette possibilité s'exprime visuellement. Dans cette étude l'affordance des référents de l'espace réel et virtuel pour l'activité de co-design est analysée, dans le sens de la possibilité de prendre action que permet la représentation visuelle.

\section{CAS D'ETUDE : L'ATELIER DE CO-DESIGN HYBRIDE}

\section{Contexte}

L'atelier observé se situe dans le cadre de la reconception d'un espace de cours existant dans le 
bâtiment de HEC-Montréal, actuellement sous-utilisé car inapproprié (Figure 1).

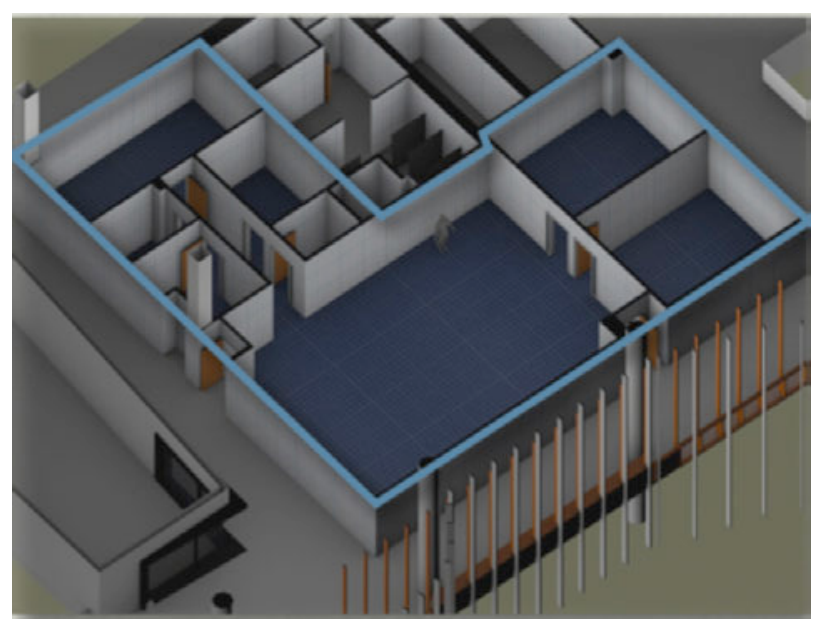

Figure 1 : Espace à reconcevoir

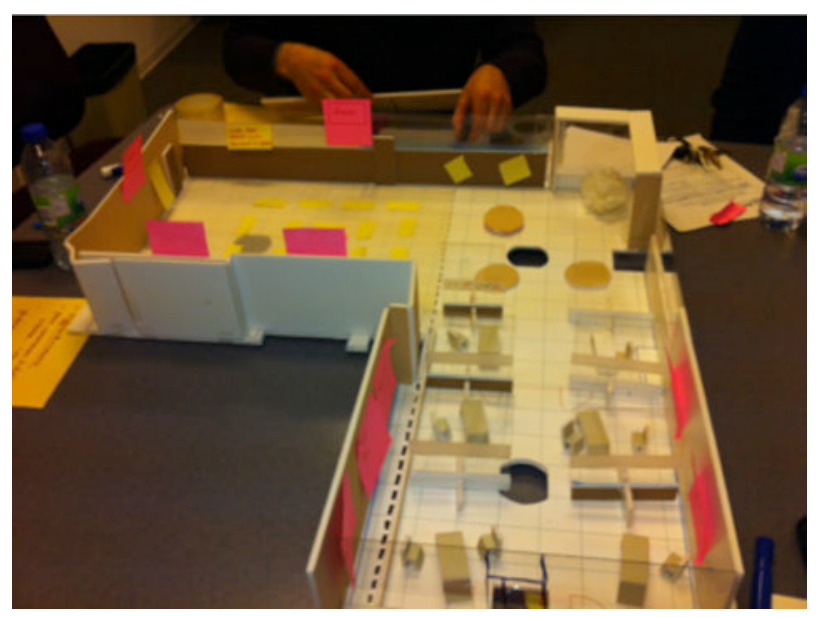

Figure 2 : une des trois maquettes finales

\section{Objectif}

L'atelier réunissait différents acteurs autour de la problématique de l'aménagement architectural d'un ensemble de classes et salles de réunion considérées comme inefficaces et technologiquement désuètes : des utilisateurs (enseignants et étudiants), des membres de la maintenance (gestion du bâtiment), des architectes responsables de la re-conception de l'espace, des designers et des facilitateurs. Au total, 24 personnes ont été réparties dans trois groupes qui avaient chacun l'objectif d'aboutir à une proposition de design pour le futur espace, dans le temps imparti d'une journée.

\section{Outils}

Différents outils ont été mis à la disposition des groupes :

- Des plans papier de la situation actuelle ;

- Des outils de dessin: papier, crayons, stylos, tableaux et craies, etc.;

- Des éléments de maquette physique : une large base sur carton rigide avec l'implantation du lieu (autour de $1 \mathrm{~m}$ par $70 \mathrm{~cm}$ ), et différents éléments (murs, fenêtre, mobilier, etc.) à assembler, le tout à l'échelle (1:25) (Figure 2);

- Un environnement immersif configuré (ouvert) qui a permis la réalité mixte : le Hybrid Ideation Space (HIS) [6] (voir plus loin) permettant aux concepteurs d'interagir dans des représentations à l'échelle réelle de l'espace à reconcevoir.

Dans le HIS, les participants étaient encadrés par un facilitateur les initiant à son fonctionnement, par conséquent aucune habileté particulière n'était requise de leur part. Spontanément les participants à l'aise en dessin manipulaient le crayon numérique du HIS, pendant que les autres concevaient, discutaient, en alternant de la représentation immersive à l'espace réel visuellement accessible du HIS (Figure 3).

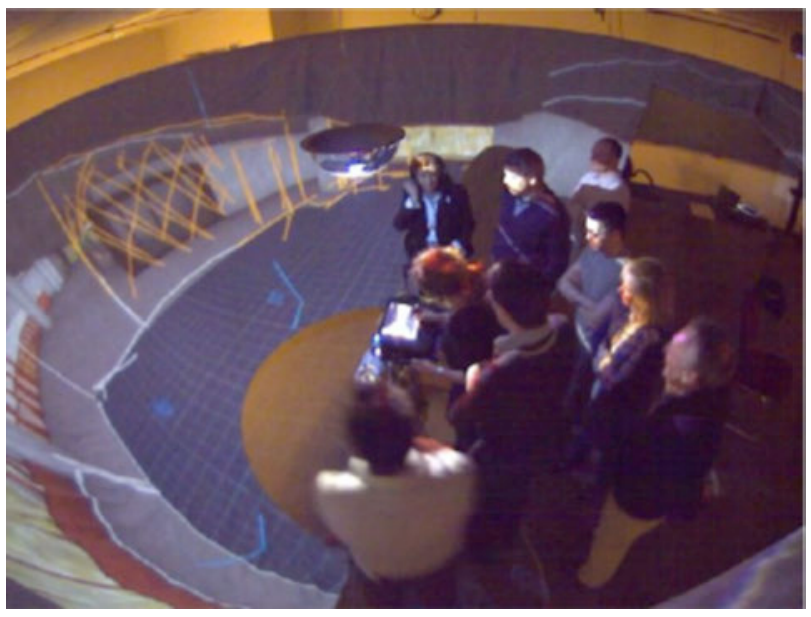

Figure 3 : Un groupe au travail dans le HIS

\section{Déroulement}

Le planning de la journée comportait quatre séances de travail d'une heure chacune, dans des locaux dédiés à chacun des groupes répartis autour de l'espace à reconcevoir (Figure 4).

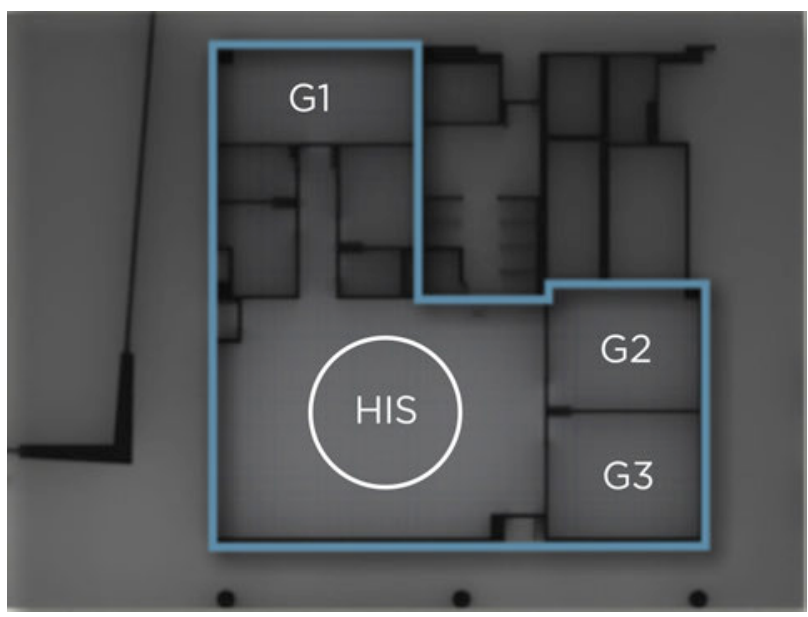

Figure 4 : organisation de l'espace de l'atelier : salles réservées aux trois groupes (G1, G2 et G3), et positionnement du HIS dans l'espace réel 
Durant chaque séance de travail, les groupes étaient invités tour à tour dans le HIS pour 20 minutes, pour concevoir au sein de l'environnement immersif. Ces séances étaient entrecoupées de pauses et de retours (théoriques) aux équipes sur leurs activités de co-design par les facilitateurs.

La particularité de cet atelier est qu'il a physiquement pris place à l'intérieur de l'actuel espace à reconcevoir. Ainsi, il nous fournit un cas d'étude spécialement intéressant pour adresser les relations entre différents modes de représentations d'un espace et la réalité physique de cet espace.

$\mathrm{Au}$ terme de la journée, des présentations formelles ont eu lieu, en utilisant des maquettes physiques et les représentations virtuelles et annotations dans le HIS comme support. Ces présentations ont abouti à la sélection collégiale du projet le plus porteur, parmi les trois solutions proposées.

\section{DISPOSITIF : HYBRID IDEATION SPACE}

Développé par le Hybridlab de l'Université de Montréal, le HIS est un environnement immersif à $360^{\circ}$ permettant de créer des représentations à échelle réelle. En rentrant dans cet environnement englobant, l'utilisateur est plongé au cœur de ses représentations graphiques (esquisses) et numériques (modèles 3D, photos, animations, etc.). Ce système utilise une procédure de projection immersive basée sur une seule projection semi-sphérique panoramique.

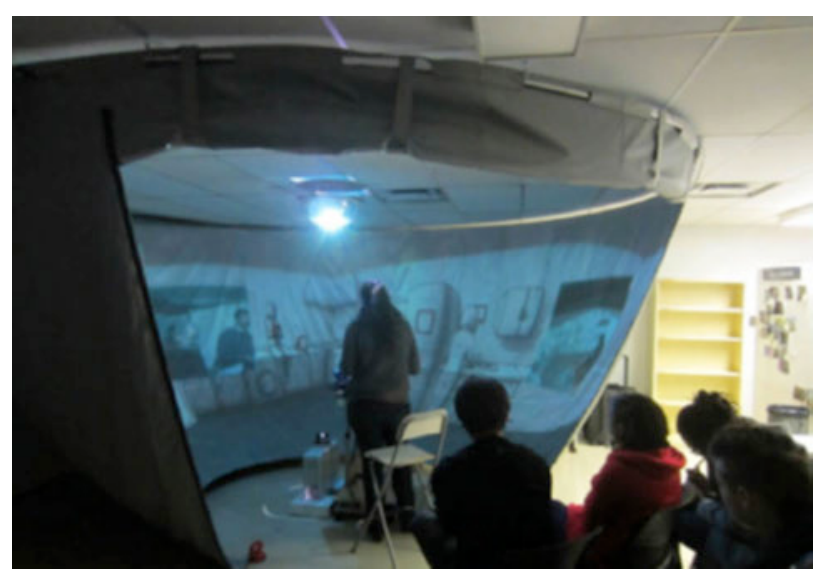

Figure 5 : HIS ouvert

Dans cet environnement dédié à la conception, les utilisateurs (jusqu'à quatre personnes une fois l'écran fermé ou un plus grand nombre avec l'écran ouvert, voir Figures 3 et 5) peuvent interagir par l'esquisse sur des représentations immersives réalisées à partir de modèles 3D et de photos. Ces représentations sont affichées sur une toile semi-sphérique autour des utilisateurs qui se trouvent immergés au sein de ces représentations. Les concepteurs peuvent dessiner dans l'environnement à main levée sur une tablette-écran (Wacom Cintiq ${ }^{\mathrm{TM}}$ 12" ici), les esquisses ainsi tracées étant affichées dans l'environnement immersif, en surimpression aux représentations importées (voir Figure 6). Il s'agit donc de créer un environnement hybride (représentations manuelles et numériques) et immersif (grâce à l'échelle réelle) pour l'idéation. Des détails sur cet environnement peuvent être trouvés notamment dans $[6,7]$.

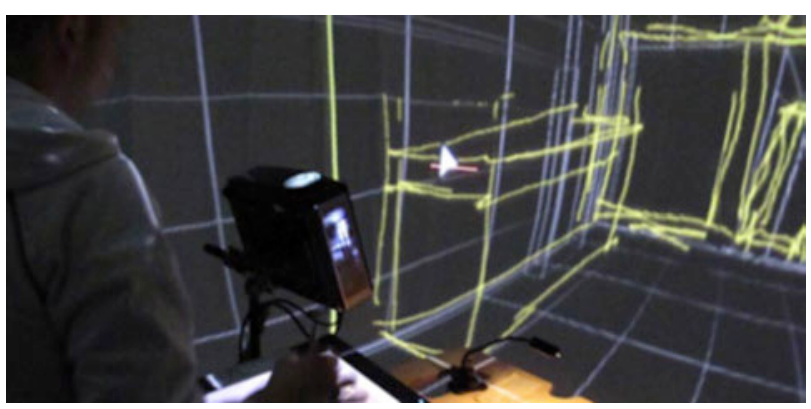

Figure 6 : dessin dans le HIS

Le HIS semble particulièrement approprié pour soutenir l'idéation individuelle et collaborative [8]. Il fournit aux utilisateurs un espace associé à un créneau temporel, tous deux dédiés à l'émergence de nouvelles idées. Le HIS, couplé aux méthodes particulières associées (le codesign dans cette étude), suscite la créativité dans les équipes de travail. Il constitue un moyen plus efficace que des environnements comparables pour l'émergence des idées [8]. Le caractère immersif permet notamment de soutenir les conversations d'idéation collaboratives, de réduire la charge mentale et de favoriser l'occurrence d'épisodes d'expérience optimale, caractéristiques de la créativité en design tel que documenté à maintes reprises [7, 8, 9]. L'expérience optimale, aussi appelée flow [4], paraît être soutenue par la qualité intuitive de l'interface du HIS (pilotée au stylo, selon des moyens naturels et sans déclaration explicite d'intention), permettant un dessin manuel augmenté par la technologie qui soutient l'action de dessin au même rythme que la conversation collaborative [8,9]. L'immersion paraît jouer un rôle aussi dans l'occurrence d'épisodes de flow, celle-ci stimulant l'activation des processus cognitifs spatiaux également associés à cet état [15].

Comme précédemment évoqué, le HIS a été installé au cœur de l'espace à reconcevoir (Figure 7). Des modèles numériques de cet espace ont été construits et affichés à l'échelle réelle dans le HIS, en immersion (Figure 8). Les participants avaient donc la possibilité de voir une projection immersive d'un modèle numérique de l'espace à concevoir, au cœur de cet espace réel, avec la possibilité d'aligner le même point de vue (voir Figure 7). Sur le modèle virtuel, il leur était aussi possible d'afficher les murs en transparence, offrant ainsi une vue vers l'extérieur de la salle. Il leur était aussi possible d'afficher d'autres vues de l'espace, par exemple une perspective extérieure à la salle. Afin de faciliter le travail collectif, le HIS a été ouvert, offrant un espace immersif de $240^{\circ}$. 


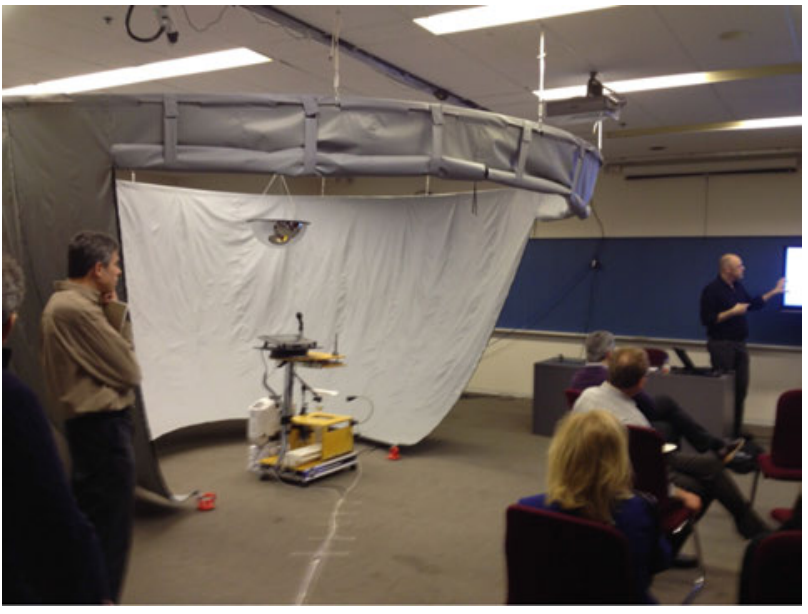

Figure 7 : HIS ouvert, au centre de l'espace à reconcevoir

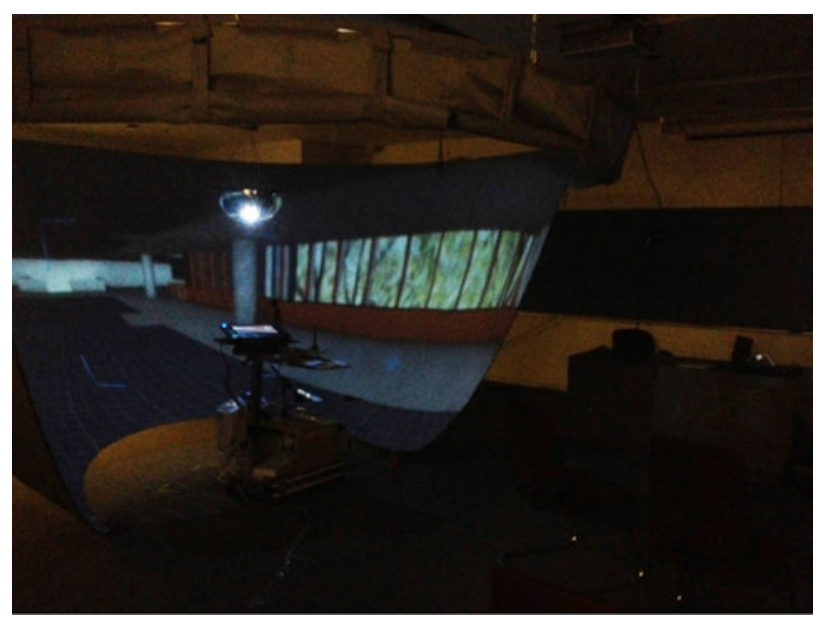

Figure 8 : représentation virtuelle alignée avec les murs en transparence

\section{QUESTIONS DE RECHERCHE}

Comme mentionnée en introduction, nous nous intéressons au rapport entre les représentations virtuelles de l'espace et la réalité tangible de ce même espace ; notamment à la façon dont les deux sont utilisées comme ressources pour la construction d'un référentiel commun. Plus spécifiquement, nous cherchons à comprendre comment les personnes en situation de conception se reposent sur le réel et la représentation virtuelle pour communiquer et échanger des idées, et comment ces ressources fournissent des affordances pour la réalisation de l'activité collective.

\section{METHODOLOGIE}

Pour ce faire, bien que l'ensemble de l'atelier ait été enregistré, nous ne traitons dans cette étude que les séances de travail dans le HIS, mobilisant les représentations virtuelles (projetées dans l'espace immersif), des esquisses immersives dans le HIS, des représentations réelles amenées par les participants lors de ces séances de travail (maquettes à l'échelle, voir Figure 9), et l'espace réel. Nous ne traitons que des séances 2,3 et 4 , la première étant la reformulation du problème de design. Le temps total des vidéos analysées est de 3 heures : 3 groupes effectuant chacun 3 sessions de 20 minutes.

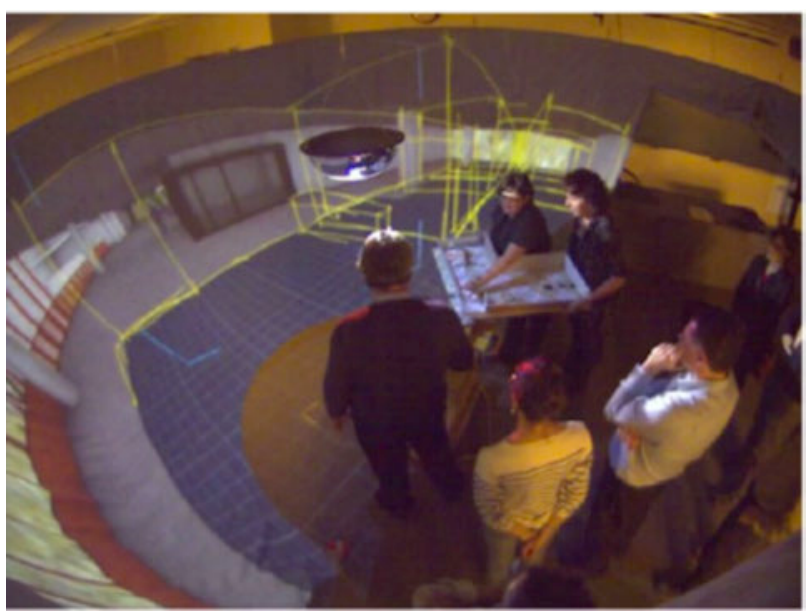

Figure 9 : maquette à l'échelle amenée dans le HIS

Pour approcher les questions qui nous occupent, notre travail concerne l'analyse des gestes de pointage effectués par les différents participants. L'idée est de comprendre comment les verbalisations des participants s'articulent avec l'utilisation de l'environnement, qu'il soit réel ou virtuel, comme ressource à la communication. Nous avons ainsi comptabilisé et codé chacun des gestes de pointage effectués lors des séances de travail sur différentes dimensions :

- La direction du mouvement: espace réel, espace virtuel ou maquette physique ;

- Le sujet de discussion de design lors du geste de pointage: la géométrie de l'espace, son ameublement, les matériaux, etc. ;

- Le contenu de la référence spatiale, qui peut concerner le situation actuelle (ex. «les enseignants aiment avoir le tableau positionné à cet endroit») ou la situation future (ex. "on pourrait mettre le tableau à cet endroit pour éviter les reflets ») ;

- Les marqueurs linguistiques des participants associés aux gestes de pointage. Ces marqueurs permettent d'évaluer le niveau de présence en situation assistée [11]. Ceux-ci ont été codés en trois catégories : ici (ici, celui-ci, à cet endroit-ci, etc.), là (là, là-bas, celui-là, etc.) et les référents explicites (au dessus de, devant, à gauche, etc.) ;

- Le rapport de configuration réel/virtuel dans le HIS au moment du geste de pointage, qui peut être de trois types (Figure 10) : (a) aligné, c'est à dire que l'orientation de l'espace virtuel projeté dans le HIS est cohérente avec l'espace réel (le point de vue subjectif sur le modèle virtuel de la salle est strictement le même que le point de vue des participants sur la salle réelle), voir aussi Figure 8; (b) non-aligné quand le modèle virtuel n'est pas orienté dans le même axe que l'espace réel ; et (c) extérieur quand un autre point de vue sur l'espace 
est proposé dans le modèle virtuel du HIS (par exemple, une vue depuis l'extérieur de la salle).

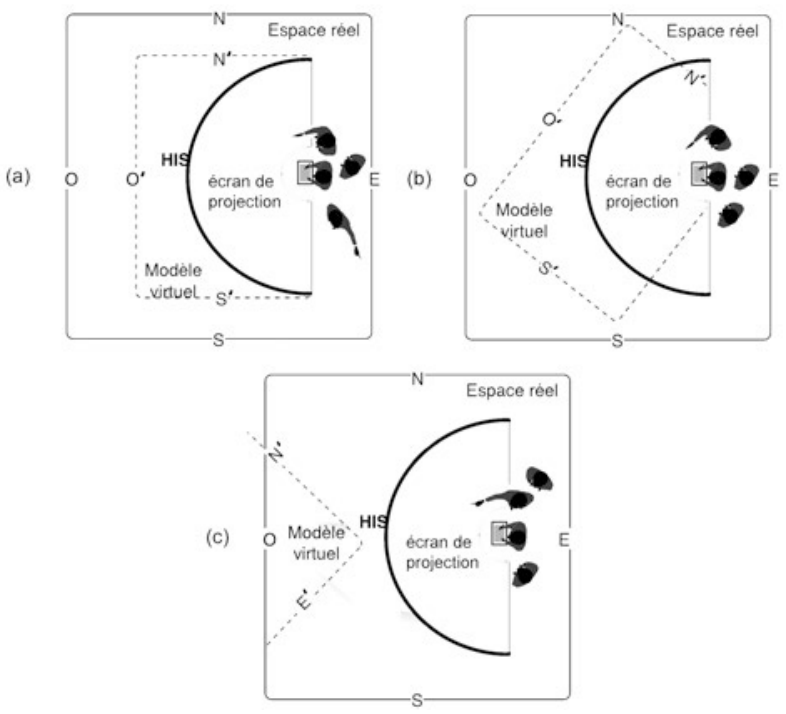

Figure 10 : Vue en plan des trois configurations possibles du rapport réel/virtuel aligné (a), non-aligné (b) et extérieur

(c). Les lettres $\mathbf{N}, \mathbf{E}, \mathrm{S}$ et $\mathrm{O}$ font référence aux murs Nord, Est, Sud et Ouest de l'espace réel, N', E', S' et O' au modèle virtuel.

Nous cherchons ainsi à comprendre sur quelles bases se reposent les utilisateurs pour concevoir une solution lors d'un travail collectif d'idéation, afin de proposer des idées et de se constituer une représentation partagée du problème. L'ensemble des gestes $(\mathrm{N}=340)$ a donc été codé sur ces différentes dimensions. Les données ont été rassemblées dans des tableaux de contingence et des proportions ont été établies, afin de saisir les tendances.

\section{OBSERVATIONS : DEROULEMENT GLOBAL DE L'ATELIER}

Avant de décrire l'usage des espaces réels et virtuels comme ressource à la communication, quelques observations d'ordre général peuvent être faites concernant le déroulement de l'atelier.

Il est d'abord à noter que les trois groupes sont arrivés à un résultat intéressant, surprenant même, au regard des objectifs initiaux. Tous les participants ont déclaré avoir passé une journée riche et intéressante, et d'avoir réellement contribué à leurs projets respectifs. Bien que cette observation soit de l'ordre du ressenti subjectif informel, et qu'elle ne puisse nous permettre de tirer des conclusions définitives, elle nous éclaire cependant sur la pertinence de la méthode de co-design mise en place, et notamment sur le dispositif représentationnel utilisé en support à ce travail de co-design (HIS et outils manuels comme la réalisation des maquettes physiques). Il apparaît qu'aussi bien le HIS que la maquette physique aient été des supports pertinents pour soutenir une conception collaborative multidisciplinaire (Figure 11).

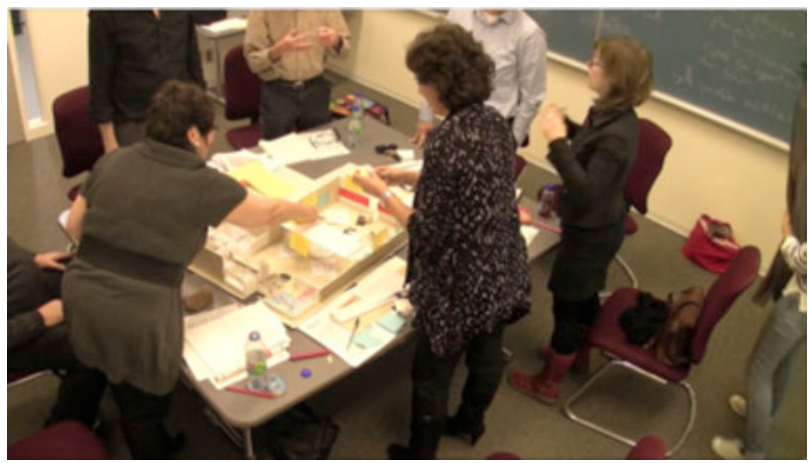

Figure 11 : co-design multidisciplinaire soutenu par les maquettes

L'usage des différents modes de représentation fait cependant l'objet d'analyses complémentaires.

Une autre observation informelle intéressante est relative à la pertinence du phénomène de réalité mixte pour la construction d'une une vision partagée du projet. Nous avons en effet pu constater que les différentes équipes, dès leur sortie du HIS, abordent directement de nouvelles problématiques de conception, sans passer par une étape de reformulation des avancées effectuées dans le HIS. Il semble donc que les équipes soient directement opérationnelles pour la conception au départ du HIS, témoignant d'une capacité de celui-ci à soutenir une vision réellement partagée du problème.

Une autre observation est aussi relative à la participation. Le HIS et la maquette physique ont tous deux été largement utilisés par l'ensemble des participants. Il s'agit donc de modes de représentation de l'espace qui s'avèrent dans une certaine mesure plus intuitifs que les modes de représentation conventionnels que sont l'esquisse en plan ou en perspective. Le rapport d'échelle directement perceptible qu'autorisent ces deux modes de représentation nous semble être un déterminant de cette efficacité perçue.

\section{RESULTATS : ANALYSE DES REFERENTS DE POINTAGE}

Résultats 1 : Développement dans le Temps des Supports aux Gestes de Pointage

Le tableau 1 montre l'évolution, au fil des réunions, des directions des gestes de pointage, c'est-à-dire des référents de la communication.

\begin{tabular}{|lrrr|}
\hline & Session 2 & Session 3 & Session 4 \\
\hline Maquette & $2 \%$ & $19 \%$ & $10 \%$ \\
Réel & $28 \%$ & $6 \%$ & $5 \%$ \\
Virtuel & $70 \%$ & $75 \%$ & $85 \%$ \\
\hline
\end{tabular}

Tableau 1. Analyse des référents de la gestuelle de pointage

$\mathrm{Au}$ fur et à mesure du processus de co-design, on constate un basculement dans les supports de la construction du référentiel commun du projet (Tableau 1). Au début (Session 2) on retrouve une bonne quantité 
de références au contexte réel (28\%), alors que les références de pointage se concentrent ensuite sur le virtuel. Au début, étant donné que la maquette n'était pas réalisée, les participants ont fait plus de références sur le réel et le virtuel (Tableau 1). Par la suite la maquette et le réel ont été utilisés pour valider certains repères.

Le tableau 2 décrit l'objet de la communication lors des gestes de pointage, selon qu'il concerne la situation actuelle ou une projection future.

\begin{tabular}{|lrrr|}
\hline & Session 2 & Session 3 & Session 4 \\
\hline $\begin{array}{l}\text { Projection } \\
\text { future }\end{array}$ & $79 \%$ & $93 \%$ & $79 \%$ \\
\hline $\begin{array}{l}\text { Situation } \\
\text { actuelle }\end{array}$ & $21 \%$ & $7 \%$ & $21 \%$ \\
\hline
\end{tabular}

Tableau 2. Analyse de contenu verbal de la conversation selon 2 modes

Sans surprise, la conversation semble évoluer entre les sessions 2 et 3 , ou les références à la situation actuelle tendent à diminuer. Si la session 2 mobilise toujours une analyse de l'existant, les comportements en session 3 témoignant d'une projection dans le futur, caractéristique des activités de design. Cependant, dans la session 4, il y a un retour sur la situation actuelle afin d'argumenter certaines propositions en vue de la présentation finale.

Notre lecture de cet atelier nous mène à croire que la représentation virtuelle était plus flexible et affordante que la maquette pour effectuer des modifications durant le processus de conception; le virtuel semble avoir servi d'espace tampon pour ensuite être représenté d'une manière permanente dans la maquette.

Résultats 2 : Analyse des Référents Propres au Design dans le Temps et selon les Supports

Quant au sujet des conversations lors de pointage, la plupart des discussions ont porté sur la géométrie de l'espace et les caractéristiques des matériaux durant toutes les sessions (Tableau 3). Il est important de remarquer que la hauteur (proportions de l'espace) n'a pas été l'objet de beaucoup de conversations (5\% au total durant les sessions), mais qu'elle a été traitée seulement à l'aide de la représentation virtuelle (Tableau 4), ce qui semble indiquer que la sensation de hauteur est suffisamment forte dans le virtuel pour n'avoir pas besoin d'être référencée dans le réel.

En dépit de la co-localisation de la représentation immersive au sein même l'espace réel à reconcevoir (réalité mixte), le tableau 4 démontre que l'ensemble des référents de design ont été traités dans le virtuel. Ceci peut s'expliquer par le fait que le virtuel ait été l'espace de choix pour explorer les possibilités de design (voir sessions 3 des Tableaux 1-2), possiblement dû au fait que la représentation virtuelle offre une meilleure affordance.

\begin{tabular}{|rrrr|}
\hline & Session 2 & Session 3 & Session 4 \\
\hline Géométrie de & $52 \%$ & $31 \%$ & $27 \%$ \\
l'espace & $0 \%$ & $1 \%$ & $4 \%$ \\
\hline Hauteur & $34 \%$ & $16 \%$ & $29 \%$ \\
\hline Matériaux & $5 \%$ & $22 \%$ & $13 \%$ \\
\hline Mobilier & $9 \%$ & $7 \%$ & $17 \%$ \\
\hline Orientation & $0 \%$ & $23 \%$ & $10 \%$ \\
\hline Ouvertures & & & \\
\hline
\end{tabular}

Tableau 3. Analyse des référents propres au design dans le temps

\begin{tabular}{|rrrr|}
\hline & Maquette & Réel & Virtuel \\
\hline Géométrie de & & & \\
l'espace & $7 \%$ & $11 \%$ & $82 \%$ \\
\hline Hauteur & $0 \%$ & $0 \%$ & $100 \%$ \\
\hline Matériaux & $12 \%$ & $18 \%$ & $70 \%$ \\
\hline Mobilier & $22 \%$ & $4 \%$ & $73 \%$ \\
\hline Orientation & $23 \%$ & $14 \%$ & $64 \%$ \\
\hline Ouvertures & $13 \%$ & $8 \%$ & $79 \%$ \\
\hline
\end{tabular}

Tableau 4. Analyse des référents propres au design selon les supports

Résultats 3 : Analyse des Gestes de Pointages et des Références Verbales en Fonction de l'Alignement de la Représentation Virtuelle par Rapport à l'Espace Réel

La représentation virtuelle pouvait être alignée ou nonalignée à l'espace réel où le HIS se trouvait. Dans une situation d'alignement, les murs du modèle virtuel de l'espace (affichés dans le HIS) sont exactement alignés avec les murs de l'espace réel qu'ils représentent. La toile du HIS est dans ce cas en quelque sorte transparente, puisque ce qui y est affiché correspond à une représentation virtuelle de ce qui se situe directement derrière la toile. Ce n'est pas le cas pour des situations non-alignées, ou le modèle virtuel n'est pas positionné en cohérence avec l'espace réel. Un positionnement extérieur à l'espace était également possible se référant aux situations où la représentation virtuelle offrait un point de vue de l'extérieur des espaces à reconcevoir, c'est-à-dire en disjonction avec l'espace réel ambiant. Ici, les participants dans le HIS voient s'afficher les murs de l'extérieur de la salle dans laquelle ils se situent. Les données du positionnement extérieur sont ici considérées comme données de contrôle puisque que cette disjonction est typique du travail traditionnel de design avec les systèmes de conception assistée par ordinateur (voir Figure 9).

Dans la situation contrôle, (positionnement extérieur), les références aux projections futures et aux situations 
actuelles sont majoritairement construites en faisant référence à l'espace virtuel, soulignant ainsi que ce support technique est suffisant pour soutenir le travail de co-design et la mise en relation avec les repères réels (Tableau 5). Lorsque l'image virtuelle était non-alignée par rapport à l'espace réel, la plupart des conversations dans le virtuel portaient sur le projet futur, ce qui apparaît normal, compte tenu que les participants concevaient directement dans cette représentation. Le réel a été utilisé plus souvent que le virtuel comme référence en parlant de la situation actuelle. Enfin, lorsque l'image virtuelle était alignée avec la réalité, c'est la représentation virtuelle qui a été essentiellement utilisée pour discuter à la fois du projet futur et des repères du présent. C'est-à-dire, que la représentation virtuelle semble être complète et facile à comprendre dans un cadre multidisciplinaire. Le réel ici semble presque disparaître ; ou est-ce le virtuel qui devient transparent ? Il existe une ambigüité dans la mesure où, compte tenu que les deux perceptions (réelle et virtuelle) sont superposées, il n'est pas évident de déterminer avec une certitude absolue quelle est la référence utilisée pour le pointage : la réalité et la virtualité peuvent à ce titre se confondre et s'interpénétrer.

\begin{tabular}{|lrr|rr|rr|}
\hline & \multicolumn{2}{l|}{ Extérieur } & \multicolumn{2}{l|}{ Non-alignée } & \multicolumn{2}{l|}{ Alignée } \\
& Future & Actuelle & Future & Actuelle & Future & Actuelle \\
Maquette & 0 & 0 & 16 & 22 & 2 & 0 \\
Réel & 4 & 8 & 10 & 43 & 10 & 20 \\
Virtuel & 96 & 92 & 74 & 35 & 88 & 80 \\
\hline
\end{tabular}

Tableau 5. Analyse des références verbales aux situations future et actuelle selon l'alignement de la représentation virtuel (en pourcentage)

\begin{tabular}{|lrrr|rrr|rrr|}
\hline & \multicolumn{3}{|l|}{ Extérieur } & \multicolumn{3}{|l|}{ Non-alignée } & \multicolumn{3}{|l|}{ Alignée } \\
& S2 & S3 & S4 & S2 & S3 & S4 & S2 & S3 & S4 \\
Maquette & 0 & 0 & 0 & 4 & 20 & 18 & 0 & 0 & 6 \\
Réel & 7 & 0 & 4 & 44 & 6 & 3 & 17 & 0 & 11 \\
Virtuel & 93 & 100 & 96 & 52 & 74 & 79 & 83 & 0 & 83 \\
\hline
\end{tabular}

Tableau 6. Analyse des pointages aux supports selon l'alignement de la représentation virtuel et en fonction du temps (en pourcentage)

Le réel reste un bon point de référence au début du projet, surtout lorsque l'image n'était pas alignée (Tableau 6). Ces données soulignent le caractère complémentaire des deux sources d'information pour soutenir le travail de co-design et ses activités connexes en début de projet (construction d'un common ground). Dans la progression du projet et tenant compte qu'il y a très peu de situations où l'image est alignée (Session 3), nous pouvons considérer la représentation virtuelle comme suffisante pour concevoir et présenter le projet de par sa prédominance dans les trois types d'alignement. Il faut quand même remarquer le fait que la maquette a été très utilisée à la fin du processus pour parler du projet lorsque le virtuel n'était pas aligné.

\section{Résultats 4 : Analyse des Marqueurs Linguistiques}

Un questionnement lié à notre approche méthodologique doit être considéré. En effet, comme évoqué plus haut, notre approche ne permet pas de distinguer clairement et objectivement la direction des références gestuelles (vers le réel ou vers le modèle virtuel) dans la situation où l'image virtuelle était alignée avec l'espace réel. Par exemple, en situation alignée, si un participant pointe vers un objet en particulier, considère-t-il l'objet réel ou virtuel ? Ceci souligne donc une difficulté méthodologique pour les futures recherches alliant virtuel et réel qui doit être résolue.

Une piste potentielle a consisté à prendre en compte des marqueurs linguistiques telles que ici et là utilisées pour évaluer le niveau de présence en situation assistée [11] entre autre par des technologies de réalité virtuelle/augmentée. En complément, nous avons considéré différentes références explicites telles que à gauche, en bas, etc.

Cette direction nous a conduit vers des résultats peu discriminants (Tableau 7). En effet, nous pouvons souligner une utilisation plus importante du là pour des références au réel (84\%). Cependant, nous ne pouvons avancer un quelconque résultat concluant quant à une utilisation distinctive du ici en lien avec le réel versus le virtuel. Néanmoins, ici semble être plus utilisé pour la maquette et le virtuel.

\begin{tabular}{|lrrr|}
\hline & Maquette & Réel & Virtuel \\
\hline ici & $48 \%$ & $12 \%$ & $36 \%$ \\
\hline là & $36 \%$ & $84 \%$ & $47 \%$ \\
\hline $\begin{array}{l}\text { Références } \\
\text { explicites }\end{array}$ & $16 \%$ & $4 \%$ & $17 \%$ \\
\hline
\end{tabular}

Tableau 7. Analyse des marqueurs linguistiques de présence en fonction des supports

Il serait donc nécessaire de rendre compte objectivement de la pertinence du marqueur ici pour la référence à un élément $x$ dans le virtuel et l'utilisation du là pour la référence à un élément $y$ dans le réel dans une prochaine analyse éventuellement en confrontant un utilisateur à ses propres références. Il se pourrait, et en tenant compte du contexte multidisciplinaire, que le ici fait référence à des pixels sur l'écran même, plutôt qu'à un référant visuel dans la représentation immersive.

\section{Résultats 5 : Énoncés Réels des Participants}

Dans la réalité, tous les gestes et leurs identifiants linguistiques étaient utilisés conjointement. Nous présentons ici un exemple d'énoncé réel de participants pour comprendre notre interprétation des résultats (voir Figure 12). Nous considérons l'exemple cité comme représentatif de l'ensemble des échanges et illustrant bien nos interprétations relatives à l'affordance de la 
représentation virtuelle et à l'utilisation du virtuel comme espace tampon lors du travail de co-design :

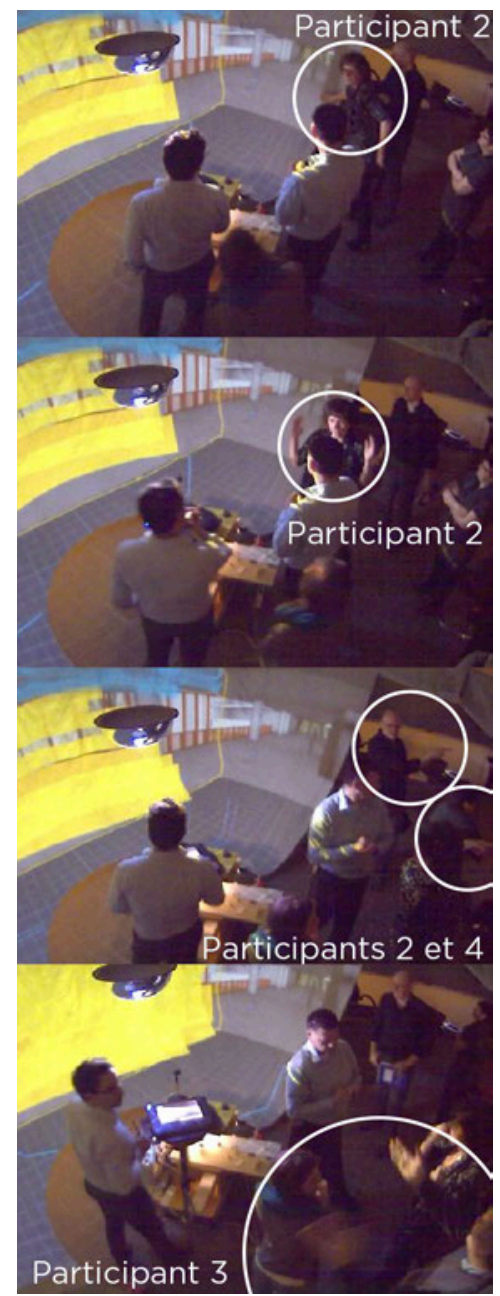

Figure 12 : Illustration des gestes accompagnants l'exemple d'énoncé réel des participants

- (Participant 1) : Il y a bien une portion de tableau... (sans geste) [Pas de codage]

- (Participant 2) : Non, non je le mettrais là (elle touche l'écran). Pour avoir une orientation de la salle dans ce sens là (elle gesticule avec ses mains vers la représentation virtuelle) c'est beaucoup mieux d'avoir une salle en long qu'avoir une salle en large. [geste: virtuel; contenu: situation future, orientation; référent linguistique : là ; config. : non-aligné]

- (Participant 1) : Le mur, en quoi ? (Il gesticule de la main vers la représentation virtuelle) en quoi? En tableau ? [geste: virtuel ; contenu : situation future, matériaux; référent linguistique : aucun ; config. : nonaligné]

- (Participants 2 et 4 pointent vers un mur de l'espace réel) : On va prendre celui-là pour le tableau. Ça me prend un écran de $T V$. [geste : réel; contenu : situation future, mobilier; référent linguistique : là ; config. : non-aligné]
- (Participant 3) : Ça prend aussi un endroit pour accrocher du papier. (Dit-il en dessinant dans l'espace vers la représentation virtuelle). [geste: virtuel; contenu: situation future, matériau; référent linguistique : aucun ; config. : non-aligné]

\section{DISCUSSION ET CONCLUSIONS}

Ce travail s'est penché sur les rapports entre les représentations virtuelles et réelles dans un contexte de réalité mixte lors d'une activité de design. Ayant profité d'une invitation à munir d'un HIS un atelier de design professionnel d'une journée, et de l'installer au centre de l'espace à reconcevoir, nous avons eu une occasion unique de voir comment les participants d'équipes multidisciplinaires réagissaient à la proximité, voir même à la fusion à certains moments des espaces physiques réels et de la représentation virtuelle immersive grandeur réelle.

En architecture ou en design d'intérieur, le travail d'idéation, avec ou sans CAO, se fait généralement en dehors du site du projet. Nous nous attendions donc à ce que les participants se concentrent principalement ou uniquement sur l'interface immersive du HIS. Or, les données collectées nous montrent que les participants font des références à la situation réelle en proportions importantes, surtout en début de processus et quand les représentations réelles et virtuelles offrent des points de vue différents et complémentaires sur le même objet (situation non-alignée). Sans surprise, avec la progression du processus de design, les références par pointage se sont concentrées sur la représentation virtuelle puisque celle-ci portait les propositions de design discutées par les groupes. A mesure que la conception sort d'une analyse de la situation existante pour s'orienter vers des projections futures de l'espace reconçu, l'espace réel perd de son importance comme support à la communication, et le modèle virtuel semble se suffire à lui-même.

Il semble aussi que l'utilisation conjointe de ressources ne soit utile que lorsque la réalité et la virtualité offrent des points de vue complémentaires du même objet. Lorsque les objets sont différents (ici, quand les designers sont dans l'espace réel alors qu'ils discutent de l'espace extérieur avec un modèle représentant l'extérieur de la pièce), l'utilisation du réel perd en importance.

Cette utilisation opportuniste de l'espace réel du projet transformant cette situation en expérience de réalité mixte suggère que la représentation virtuelle grandeur réelle répondait aux attentes situées (embodied) des participants, l'aller-retour entre les deux types d'espaces corroborant une réalité complémentaire, référant ici aux principes d'Anders [1].

La représentation virtuelle offrant la possibilité de poser une action de design (affordance) fut préférée à l'espace réel, d'une manière générale mais principalement lorsque 
les propositions de design deviennent abouties et nécessitent l'utilisation de modèles.

Cela suggère que la construction de l'image mentale dans la transition du réel au virtuel a été facile, voir complémentaire. Une fois le processus de design bien enclenché, le virtuel semble avoir répondu aux attentes complètement. La représentation virtuelle semblait suffisamment crédible pour que les participants s'en servent sans devoir s'appuyer ou aller vérifier dans le réel. La réalité mixte offre une continuité du réel au virtuel. C'est pourquoi le réel disparaît surtout en situation alignée.

\section{REMERCIEMENTS}

Cette communication est subventionnée par le projet «Co-Design augmenté : Transparence et extraversion de l'idéation à travers le HIS interconnecté », Subvention Savoir, CRSH et par le projet Actions de Recherches Concertées COMMON - Académie Wallonie Europe, Communauté Française de Belgique. Nous voulons remercier la MATI-Montréal, les membres du groupe Collaborons.ca, le HEC Montréal et tous les participants et assistants de recherche pour leur aide et support à cette étude.

\section{BIBLIOGRAPHIE}

1. Anders, P. Cybrid principles: Guidelines for merging physical and cyberspaces. International Journal of Architectural Computing, 3 (3), (2005), 391-406.

2. Azuma, R.T. A Survey of Augmented Reality. Presence: Teleoperators and Virtual Environments, 6(4), (1997), 355-385.

3. Bilda, Z. et Gero, JS. Does sketching off-load visuospatial working memory? in Gero, JS et Bonnardel, N (eds), Studying Designers '05, Design Computing and Cognition, (2005), 145-159.

4. Csikszentmihalyi, M. et I. S. Csikszentmihalyi. Optimal experience, psychological studies of flow in consciousness. Cambridge University Press, (1988).

5. Détienne, F., Boujut, J.-F., et Hohmann, B. Characterization of collaborative design and interaction management activities in a distant engineering design situation. COOP 2004, (2004), 83-98.

6. Dorta, T. Implementing and Assessing the Hybrid Ideation Space: a Cognitive Artifact for Conceptual Design. International Journal of Design Sciences and Technology, 14 (2), (2007), 119-133.

7. Dorta T., Lesage A. et Di Bartolo C. Collaboration and design education through the interconnected HIS:
Immature vs. Mature CI Loops observed through Ethnography by Telepresence. eCAADe 2012 : Physical Digitality, 2, (2012), 97-105.

8. Dorta T., Kalay Y., Lesage A. et Pérez E. Comparing immersion in collaborative ideation through design conversations, workload and expérience. Integration Through Computation, ACADIA 2011, (2011), 216225.

9. Dorta, T., Pérez, E. and Lesage, A. The Ideation Gap: Hybrid tools, Design flow and Practice. Design Studies. 29 (2), (2008), 121-141.

10.Dubois, E. Chirurgie Augmentée, un Cas de Réalité Augmentée; Conception et Réalisation Centrées sur l'Utilisateur. Thèse de doctorat, Université Joseph Fourier, Grenoble I, (2001)

11.Fussell, S. R., Kraut, R.E. et Siegel, J. Coordination of communication: effects of shared visual context on collaborative work. CSCW 2002, (2002), 21-30.

12. Gibson, J. The Ecological Approach to Visual Perception. Lawrence Erlbaum, (1979).

13. Gibson, J. The concept of affordances. Perceiving, acting, \& knowing, (1977), 67-82.

14.Lebahar, J.-C. Le dessin d'architecte. Simulation graphique et réduction d'incertitude. Editions Parenthèses, (1983).

15.Lesage A. et Dorta T. Beyond the functional / pleasurable split : User Experience with Conceptual Design Tools. Proc. CAADRIA 2011, (2011), 291300 .

16. McGrenere, J., et Ho, W. Affordances: Clarifying and evolving a concept. Graphics Interface, (2000), 179186.

17. Milgram, P. et Kishino, F. A Taxonomy of Mixed Reality Visual Displays. IEICE Transactions on Information Systems, E77(12), (1994).

18. Norman, D. A. The psychology of everyday things. Basic Books, (1988).

19. Norman, D. A. Affordance, conventions, and design. Interactions 6(3), (1999), 38-43.

20.Passini, R. Spatial representations, a wayfinding perspective. Journal of Environmental Psychology, 4 (2), (1984), 153-164.

21. Van de Vreken, A., et Safin, S. Influence du type de représentation visuelle sur l'évaluation de l'ambiance d'un espace architectural. IHM10 : Conférence francophone sur l'interaction homme-machine. Luxembourg, (2010), pp. 49-56 